\section{Cheilitis Associated With Sensitization to Penicillium notatum in a Clarinetist}

Jaqueti $\mathrm{P}^{1}$, García $\mathrm{MI}^{2}$, Campanón-Toro $\mathrm{MV}^{1}$, Sobrino $\mathrm{M}^{1}$, Gallardo A ${ }^{1}$, Dávila I ${ }^{3}$

${ }^{1}$ Allergy Service, University Hospital of Salamanca, Salamanca, Spain

${ }^{2}$ Microbiology Service, University Hospital of Salamanca and Institute for Biomedical Research of Salamanca (IBSAL), Salamanca, Spain

${ }^{3}$ Allergy Service, University Hospital of Salamanca and Institute for Biomedical Research of Salamanca (IBSAL), Salamanca, Spain; Department of Biomedical and Diagnostic Sciences, Salamanca University School of Medicine, Salamanca, Spain

J Investig Allergol Clin Immunol 2020; Vol. 30(4): 292-293 doi: 10.18176/jiaci.0494

Key words: Cheilitis. Penicillium notatum. Contact dermatitis. Clarinet. Atopy.

Palabras clave: Queilitis. Penicillium notatum. Dermatitis de contacto. Clarinete. Atopia.

Cheilitis is an inflammatory process affecting the lips. It could be due to various causes, such as extreme temperatures, malignant conditions (actinic cheilitis), nutritional deficiencies, infections, atopic dermatitis, and contact dermatitis [1]. Isolated cases of cheilitis due to contact dermatitis caused by sensitization to wood have been reported in wind instrument players [2-5]. Allergic contact dermatitis is the most frequently reported allergic condition affecting violinists and violists [6].

A 15-year-old boy consulted in 2017 for recurrent episodes of cheilitis. He had been treated with repeated cycles of a potent topical corticosteroid (clobetasol), and his clinical condition improved. Nevertheless, cheilitis relapsed when the treatment was stopped. He also reported that several months previously, he had experienced a self-limiting episode of labial angioedema while eating a pork loin sandwich. When specifically asked about his hobbies, he told us that he had played the clarinet since the age of 8 years using a wooden mouthpiece (Arundo donax). He also reported a personal history of allergic rhinoconjunctivitis due to sensitization to the fungus Penicillium and had rhinoconjunctivitis that was related to exposure to a fish tank in his bedroom. His symptoms resolved when the fish tank was removed. He had also been diagnosed with allergic rhinoconjunctivitis due to grass pollen allergy that had improved notably after 4 years of sublingual immunotherapy with a 5-grass extract. The physical examination was unremarkable, except for upper and lower lip cheilitis (Figure).

Skin prick tests with a locally adapted battery of aeroallergens were positive to Penicillium species and grass pollen. Specific IgE results (ImmunoCAP, Thermo Fisher Scientific) were as follows: Penicillium notatum $(7.13 \mathrm{kU} / \mathrm{L}$

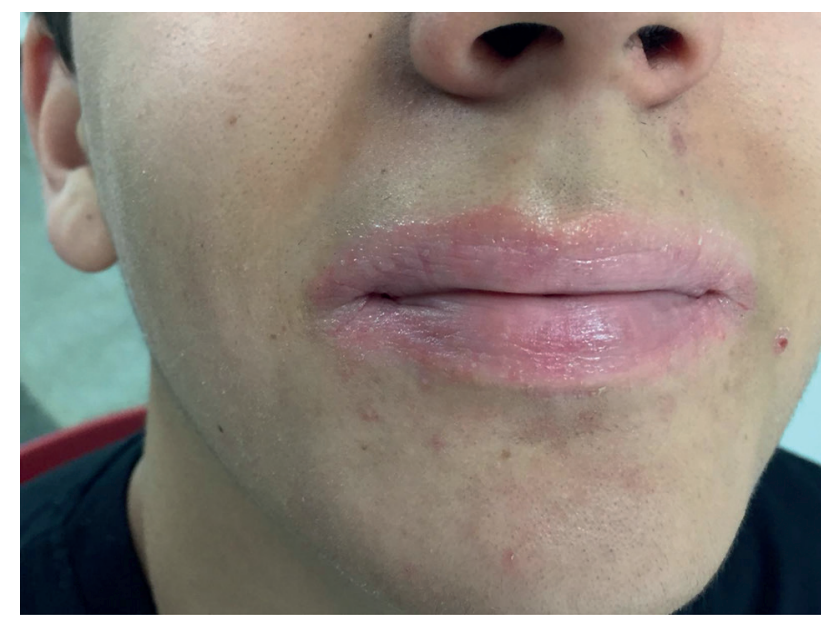

Figure. Upper and lower lip cheilitis.

and $13.4 \mathrm{kU}_{\mathrm{A}} / \mathrm{L}, 5$ years ago and at present, respectively) and Phleum pratense $(65.1 \mathrm{kU} / \mathrm{L})$. Total $\mathrm{IgE}$ was $357 \mathrm{kU} / \mathrm{L}$.

Culture of the mouthpiece was performed by the Department of Medical Microbiology on blood agar plates and Sabouraud dextrose agar with chloramphenicol for the selective isolation of fungi. A fungus had grown at 48 hours and was identified as $P$ notatum using matrix-assisted laserdesorption ionization-time-of-flight mass spectrometry [10]. The microbiologist did not know that the patient was sensitized to $P$ notatum.

We advised the patient to change the mouthpiece for a plastic one and to wash it with a disinfectant solution after use. At his 1-year check-up, the patient reported that he had not experienced any further episodes of cheilitis.

We present the case of an atopic clarinet player who developed recurrent episodes of cheilitis. He had previously experienced rhinoconjunctivitis due to $P$ notatum. We were able to demonstrate the growth of $P$ notatum in the wooden mouthpieces that he used when playing the clarinet. Cheilitis due to contact dermatitis caused by the wood used in wind instruments has been reported by several authors [2-5]. Ruiz Hornillos et al [2] and McFadden et al [3] both reported a case of cheilitis in a clarinetist who used a cane reed nozzle. Inoue et al [4] reported a case with similar symptoms in a saxophonist, also due to a cane reed mouthpiece. None of those patients were sensitized to molds. Van der WegenKeijser et al [5] reported cheilitis in a saxophonist, although the mycological culture of the nozzle was negative for molds. In the present case, we were able to rule out the wood of the mouthpiece as the culprit factor because the patient had later been playing the same clarinet using a plastic mouthpiece that he disinfected after every use without relapse of cheilitis.

Concerning the mechanism of the reaction, it is clear that the fungus $P$ notatum was present in the mouthpiece. It seems that the organic nature of the mouthpiece, together with the humidity provided by the saliva, provides a favorable substrate for the growth of the fungus. Nevertheless, we cannot say whether an IgE-mediated mechanism (protein contact dermatitis, as the patient was sensitized to $P$ notatum, demonstrated by skin prick test and sIgE) or a type IV contact 
mechanism is involved (as in other case reports with similar symptoms due to type IV sensitization to woods) [2-7]. Finally, the episode of lip angioedema when eating pork loin could be explained by the fact that processed cold meat is stuffed into casing with mold cultures to enhance flavor and aroma [8-9].

To the best of our knowledge, we report the first case of cheilitis due to $P$ notatum.

\section{Funding}

The authors declare that no funding was received for the present study.

\section{Conflicts of Interest}

The authors declare that they have no conflicts of interest.

\section{References}

1. Pilipović K, Crnarić I, Šitum M, Duvančić T. Differential Diagnosis of Cheilitis - How to Classify Cheilitis? Acta Clin Croat. 2018;57:342-51.

2. Ruiz-Hornillos JF, Alonso E, Zapatero L, Pérez C, MartínezMolero I. Clarinetist's cheilitis caused by immediate-type allergy to cane reed. Contact Dermatitis. 2007;56:243-5.

3. McFadden JP, Ingram MJ, Rycroft RJG. Contact allergy to cane reed in a clarinetist. Contact Dermatitis. 1992;37:117.

4. Inoue A, Shoji A, Yashiro K. Saxophonist's cane reed cheilitis. Contact Dermatitis. 1998;39:37.

5. Van der Wegen-Keijser MH, Bruynzeel DP. Allergy to cane reed in a saxophonist. Contact Dermatitis. 1991;25:268-9.

6. Gambichler T, Boms S, Freitag M. Skin Conditions in Instrumental Musicians: A Self-Reported Survey. BMC Dermatol. 2004;4:3-15.

7. Krenitsky A, Ramsauer K, Hossler E, Mowad C. Allergic contact dermatitis following occupational exposure to various exotic and domestic woods. Contact Dermatitis. 2019;81:1-3.

8. Brito FF, Mur P, Leal JA, Galindo PA, Gómez E, Borja J, et al. Penicillium nalgiovense as an occupational and contact allergen. J Allergy Clin Immunol. 2003;112:213-5.

9. Wantke F, Simon-Nobbe B, Pöll V, Götz M, Jarisch R, Hemmer W. Contact dermatitis caused by salami skin. Contact Dermatitis. 2011;64:111-4.

10. Sanguinetti M, Posteraro B. Identification of molds by matrixassisted laser desorption ionization-time of flight mass spectrometry. J Clin Microbiol. 2017;55:369-79. https://doi. org/10.1128/JCM.01640-16.

- Manuscript received February 3, 2020; accepted for publication February 13, 2020.

M Valle Campanón-Toro

Servicio de Alergia, Complejo Asistencial Universitario de Salamanca

Paseo de San Vicente, 58 37007 Salamanca, Spain

E-mail: mvallect@gmail.com 